

\title{
Editorial
}

\section{Moving closer to paramedic ACS rule-out in the field}

\author{
Marc Colbeck CCP, $\mathrm{PhD}$ (c) is a Critical Care Paramedic, and Senior Lecturer and Course Coordinator ${ }^{1}$. He is former \\ General Manager of Clinical Governance, South Australia Ambulance Service
}

\author{
Affiliations: \\ ${ }^{1}$ Australian Catholic University, Brisbane, Queensland
}

Almost 30 years ago my colleagues and I entered into pre-hospital care equipped with a treatment regimen that we referred to at the time as 'BOB' - bed, oxygen, blanket. We carried no medications other than oxygen, we could not insert an intravenous cannula, and we did not carry a monitor/defibrillator. Yet we were routinely called upon to assess and care for patients potentially experiencing an acute myocardial infarction.

Recognition of the patient with acute coronary syndrome (ACS) is a diagnostic challenge not only for modern paramedics, but also for emergency medicine physicians in well-equipped, modern hospital emergency departments (EDs) (1). Chest pain patients make up a low volume, high risk cohort. With an increasing mandate to keep low acuity patients out of hospital, paramedics play an important role in determining whether the patient with vague thoracic complaints and an ambiguous history should be taken to an ED, or to their local general practitioner.

Paramedics have historically been taught to rely on the patient history and presentation, including the 12-lead electrocardiogram (ECG), to make this decision. Likelihood ratios are used to describe a tool's ability to rule-in or rule-out a specific diagnosis, and any likelihood ratio score greater than 10 is considered sufficient to 'rule in' a condition (2). Even though the likelihood ratio of 12-lead ECGs to rule in ACS is 23, ECGs are complex and prone to misinterpretation. Likewise, the pre-hospital 12-lead ECG often differs from that taken in the hospital ED, creating some additional discussion. Clinicians most commonly scrutinise the 12-lead ECG for signs of ST segment elevation, which suggests ACS, yet the most common cause of ST elevation is not ACS (3). Furthermore, less than half of patients with ACS have clear diagnostic changes on their first 12-lead ECG, and roughly $10 \%$ never show any electrocardiographically indicative changes (4).

The ideal test to rule-out ACS in the field would be a simple point-of-care test - equivalent to that used in hospitals - that is one part of an evidence-based assessment protocol evaluated for appropriate sensitivity and specificity in the paramedic setting. Historically, such a validated tool has not existed, and woe-betide the naïve paramedic who decided they could 'rule-out' ACS in the field, leaving behind a patient with chest pain. This was often the first step in clinical misadventure, or at the very least, a vibrant discussion with their medical director or clinical manager.

However, this ideal is now becoming a reality. Fifth generation, high-sensitivity troponin testing, which is a simple yes/no test that offers a likelihood ratio for ACS of 59, is now available in the field. The Roche cobas h232 is one point-of-care device that paramedics can use to perform this evaluation, and it does so in less than 12 minutes. A comprehensive and evidence-based assessment protocol (the 'RSVP3 HEART Exam'), which includes 12-lead ECG and troponin, was presented recently in this journal (5) and that paper ended by recommending further testing of the HEART Exam (including troponin testing) on prehospital patients.

Shortly after, the European Journal of Acute Cardiovascular Care fortuitously published exactly such a study (6) referred to as the 'FamouS Triage' study.

This study consecutively enrolled 1426 male or non-pregnant female patients experiencing chest pain suggestive of acute myocardial infarction without STE, as these are our 'ambiguous' chest pain patients. Blood was drawn and transported with each patient to hospital. On arrival, the blood was tested for troponin using fifth generation high sensitivity troponin testing equipment in the hospital laboratory. 
Roughly $36 \%$ of the patients in the study ( $N=403$ ) had a HEART score of $0-3$; suggesting they were not experiencing ACS. Of those, only five patients had an elevated troponin value based of the pre-hospital blood sample. However, none of the five patients had ACS, or any other major adverse cardiac event in the month following their evaluation. Thirty-three of the 403 patients with a HEART score of 0-3 were admitted to hospital, of which only two required urgent clinical management (one for pulmonary embolism and one for acute pancreatitis). Both patients would have been sufficiently symptomatic that they would have been transported to hospital regardless of their HEART score, 12-lead ECG or troponin values.

This study's usefulness for ruling out ACS in the field was limited by the fact that the high sensitivity troponin testing was done following the patient's arrival at hospital. However, point-of-care fifth generation, high-sensitivity cardiac troponin testing can now be performed in the field. The elements of the ideal test to rule out ACS in the field are now in existence and available to paramedics.

The FamouS Triage authors concluded it seems feasible to rule out safely an AMI in a pre-hospital setting using the modified HEART score at the home of patients with chest pain without ST-segment elevation on their ECG'. In their next study, they plan to evaluate troponin in the field.

As the study results are disseminated we are likely to move closer to the point where paramedics managing patients with chest pain and no STE on the 12-lead ECG could call for intensive care paramedics to come and assess their patient and within 20 minutes be able to definitively rule-out ACS and, if clinically appropriate, leave the patient at home for low-acuity follow up with their general practitioner. This would offer an advantage to not only individual patients, but to the entire enterprise of acute health care as well.

Marc Colbeck

Australian Catholic University, Queensland

\section{References}

1. Lee CP, Uk M, Medicine Ml, et al. Advances emergency physician estimates of the probability of acute coronary syndrome in a cohort of patients enrolled in a study of coronary computed tomographic angiography. CJEM 2012;14:147-56.

2. Grimes DA, Schulz KF. Refining clinical diagnosis with likelihood ratios. Lancet 2005;365:1500-5.

3. Brady WJ, Perron AD, Martin ML, Beagle C, Aufderheide TP. Cause of ST segment abnormality in ED chest pain patients. Am J Emerg Med 2001;19:25-8.

4. Morris F, Brady WJ. Acute myocardial infarction - Part I. BMJ 2002;324:831 -34.

5. Colbeck M. Paramedic diagnosis of acute coronary syndrome in the out-of-hospital patient with acute, non-traumatic chest pain: The RSVP3 HEART Exam. Australasian Journal of Paramedicine 2016;13(4).

6. Ishak M, Ali D, Fokkert MJ, et al. Fast assessment and management of chest pain patients without ST-elevation in the prehospital gateway (FamouS Triage): ruling out a myocardial infarction at home with the modified HEART score. Eur Heart J Acute Cardiovasc Care 2017. [EPub ahead of print]. DOI: 10.1177/2048872616687116. 